The color red reduces snack food and soft drink intake

$$
\text { Oliver Genschow }^{\mathrm{a}, \mathrm{b}} \text {, Leonie Reutner }{ }^{\mathrm{a}} \text {, Michaela Wänke }{ }^{\mathrm{b}}
$$

${ }^{a}$ University of Basel, Department of Social and Economic Psychology, Missionsstrasse 62A, 4055 Basel, Switzerland

${ }^{\mathrm{b}}$ University of Mannheim, Center of Consumer Psychology, Parkring 47, 68159

Mannheim, Germany

\title{
published in Appetite
}

\author{
Author Note:
}

First and second authors contributed equally. Order was determined alphabetically.

E-mail addresses: oliver.genschow@unibas.ch, leonie.reutner@unibas.ch, michaela.waenke@uni-mannheim.de 


\begin{abstract}
Based on evidence that the color red elicits avoidance motivation across contexts (Mehta \& Zhu, 2009), two studies investigated the effect of the color red on snack food and soft drink consumption. In line with our hypothesis, participants drank less from a red labeled cup than from a blue labeled cup (Study 1), and ate less snack food from a red plate than from a blue or white plate (Study 2). The results suggest that red functions as a subtle stop signal that works outside of focused awareness and thereby reduces incidental food and drink intake.
\end{abstract}


The color red reduces snack food and soft drink intake

The widespread availability and ubiquity of food products causes serious health problems in industrialized societies. In the U.S. for example, the age-adjusted prevalence of obesity (BMI $\geq 30$ ) in 2008 was higher than $33 \%$ (Flegal, Carroll, Ogden, \& Curtin, 2010). Moreover, the intake of snacks (e.g., salty snacks and sweetened beverages) and the contribution of snacks to total energy intake has steadily increased during the past decades (Piernas \& Popkin, 2010). One cause for this alarming development may be the difficulty of restraining food intake. Indeed, research has shown that a conscious decision to stop eating or drinking requires self-control and mental resources (Herman \& Polivy, 2004). When resources are low, self-control fails and over-eating is likely. This effect is even intensified when snacking and drinking occur incidentally and outside of focused attention, and thereby without much self-monitoring (Tuomisto, Tuomisto, Hetherington, \& Lappalainen, 1998; Wansink, 2004).

Moreover, in situations where consumption occurs on a more habitual level and therefore outside of focused attention, consumption behavior is more susceptible to situational cues than conscious intentions (for a review, see van't Riet, Sijtsema, Dagevos, De Bruijn, 2011). For example, people adjust their food intake according to other people's food intake (Pilner \& Mann, 2004), the size of food packaging (Rolls, Roe, Kral, Meengs, \& Wall, 2004) or the size of food portions (Wansink, Painter, \& North, 2005). Because of the susceptibility to situational cues in situations where snacking occurs incidentally, situational cues that trigger a "stop" reaction may be highly beneficial in order to avoid over-consumption. We propose that the color red may function as such an unobtrusive consumption-stopping cue.

As Elliot and Maier (2007) argue, color “communicates specific information” (p. 251) but its influence is likely to occur outside of conscious awareness. Color specific associations may be culturally learned or grounded in biology or both. Due to its high 
contrast against most other colors occurring in natural environments, the color red is easily detectable. It is thereby naturally predisposed as a signal for warning as many examples from nature (see below) demonstrate. Likewise, in man-made environments red is often used for signaling danger, prohibition, and the need for vigilance (e.g., red traffic lights, stop-signs, red alert). Both, biological predispositions and culturally learned associations, may have contributed to the fact that red seems to induce a general avoidance motivation ${ }^{1}$ (Elliot, Maier, Binser, Friedman, \& Pekrun, 2009; Mehta \& Zhu, 2009). For example, a red background facilitated solving anagrams of avoidance-related words (e.g. prevent) and impeded solving anagrams of approach-related words (e.g. advance) compared to a blue background (Mehta \& Zhu, 2009). Other evidence that red induces avoidance comes from findings that red color cues on a computer screen prompted research participants to move their body away from the screen to a greater degree than did grey or green cues (Elliot et al., 2009). In addition, a number of studies found red to induce avoidance motivation or behavior in more applied and consumer oriented contexts (e.g., Belizzi \& Hite, 1992; Gerend \& Sias, 2009; Mehta \& Zhu, 2009). For example, in a simulated purchase, Bellizzi and Hite (1992) found that in red, compared to blue environments, people made less purchases, more purchase postponements, and showed weaker inclinations to shop and browse.

More central for our hypothesis, evidence from biology suggests that red may elicit avoidance with regard to food intake. Several animals with an effective chemical protective mechanism are often red colored (e.g., the firebellied toad, the lady beetle, the burnet moth) and thereby signal warning and inedibility towards their potential predators, which in turn respond attentively and cautiously. Importantly, even in non-human species this association of red and food avoidance is not restricted to the color of the target but generalizes to the color of environmental cues. For example, male rhesus macaques' behavior towards food is influenced by the color of their keepers' shirt. When having the 
chance to steal food from different keepers, macaques avoid keepers who are wearing red, compared to those wearing blue or green (Khan, Levine, Dobson, \& Kralik, 2011).

Taken together, these findings suggest that red induces avoidance behavior across contexts and across species. Because of the avoidance inducing effect of red in general and the avoidance inducing effect of red among non-human species in a food context in specific, we hypothesize that red elicits avoidance behavior towards food and beverages in humans as well.

Because snacking may contribute substantially to the increase in obesity rates, we were particularly interested in the influence of color on incidental consumption of rather unhealthy food and beverages (i.e., salty snacks and sugared soft drinks) that is not primarily driven by physiological needs such as thirst or hunger. We assumed that the color red could function as a situational cue signaling "stop" and would hence interfere with snack and soft drink consumption compared with other colors (e.g., blue or white).

\section{Overview of Research}

To test our prediction we conducted two studies. In Study 1 we tested whether blue and red stickers on drinking cups affected participants' soft drink intake. We assumed participants would drink less from red than from blue labeled cups. In Study 2 we tested the impact of subtle color cues on eating behavior and offered participants salty snack food from a red, a blue, or a white plate. Again, we assumed that red would reduce snack food intake.

\section{Study 1}

\section{Method}

Participants and Design. In total, 41 male students took part in our study $\left(M_{\text {age }}=22.61, S D=6.45\right)$ and were randomly assigned to one of the two color conditions (red label vs. blue label). A pretest with unlabeled cups had revealed that many female 
participants took only tiny sips of each beverage, and on average, females drank only half as much as males. We therefore restricted the first study to male participants only.

Procedure and Materials. In the entrance hall of the university, participants were approached and asked to test and evaluate three different soft drinks. Because we were interested in drinking behavior that was not driven by thirst, we excluded persons who reported to have consumed more than three alcoholic drinks within 24 hours prior to the inquiry from participating in the study, because of a potentially increased thirst level.

To further ensure that participants amount of drink intake would not be driven by thirst, the experimenter asked participants to drink from a $300 \mathrm{ml}$ plastic cup of water until they were not thirsty anymore. Participants then received three slightly yellowish soft drinks of different flavors (white tea, lemon, and green tea). Participants were not informed about the flavors of the drinks. The soft drinks were served in clear $300 \mathrm{ml}$ plastic cups each filled with about $200 \mathrm{ml}$ of beverage and were labeled in white color with the letters A, B, or C. The background color of all three labels was either red in one condition or blue in the other condition. Color was manipulated according to the parameters of the HSL schema reported by Mehta and Zhu (2009; red: hue $=0$, saturation $=240$, lightness $=120$; blue: hue $=160$, saturation $=240$, lightness $=120$ ). Thereby, saturation and lightness was kept constant in both color conditions. The position of the three drinks was constant with drink A (white tea flavored) always on the left, drink B (lemon flavored) in the middle, and drink C (green tea flavored) on the right; but participants were allowed to decide for themselves in which order to test the drinks. After participants had finished testing the drinks, they evaluated the beverages as part of the cover-story on three items ("I like this drink"; "The drink appeals to me"; "I would recommend this drink to a friend") on 9-point scales $(1=$ not at all; 9 = very $m u c h)$. Finally, participants completed a questionnaire containing demographic items, were probed for suspicion, debriefed and dismissed. In 
order to assess the actual variable of interest (i.e. amount consumed), each cup was weighed before and after consumption.

\section{Results and Discussion}

None of the participants correctly guessed our hypothesis or mentioned color as part of our experiment. To test our hypothesis, we first computed the mean composite of drink intake for all three drinks. A one-way ANOVA with condition (red labeled vs. blue labeled drinks) as a factor revealed that overall participants drank significantly less when the cups had red labels compared to blue labels, $F(1,39)=4.52, p=.04, \eta_{\mathrm{p}}^{2}=.10$.

Figure 1 shows that for all three beverages, drink intake was higher for blue $(M=57.24$, $S D=41.74)$ than for red labeled cups $(M=33.59, S D=22.21)$, with a significant difference between conditions for beverage A $\left(M_{\text {red }}=39.76, S D=22.02 ; M_{\text {blue }}=72.50, S D\right.$ $=62.99), F(1,39)=4.20, p=.05, \eta_{\mathrm{p}}^{2}=.10$, and beverage $\mathrm{B}\left(M_{\mathrm{red}}=24.47, S D=17.53\right.$; $\left.M_{\text {blue }}=39.67, S D=27.21\right), F(1,39)=4.08, p=.05, \eta_{\mathrm{p}}{ }^{2}=.10$, whereas the difference for beverage $\mathrm{C}$ did not reach conventional levels of significance $\left(M_{\mathrm{red}}=36.53, S D=32.09\right.$; $\left.M_{\text {blue }}=59.54, S D=52.47\right), F(1,39)=2.57, p=.12, \eta_{\mathrm{p}}^{2}=.06$. We take this as first evidence that color cues associated with avoidance relative to those associated with approach motivation may influence actual consumption behavior - in this case the amount of soft drink consumption.

In a further analysis we also tested whether color had an effect on the evaluation of the drinks. For each beverage the three evaluation items were combined into a single score reflecting the drinks' appeal (Cronbachs's alpha $=.90)$. A two-way mixed ANOVA was conducted with condition (red labeled vs. blue labeled) as the between-subjects variable and beverage (A vs. B vs. C) as the within-subjects variable. The analysis revealed that the beverages differed in their appeal, $F(2,38)=12.82, p<.001, \eta_{\mathrm{p}}{ }^{2}=.25$, with beverage A being liked most $(M=5.37, S D=1.72)$, beverage B being liked least $(M=$ $3.24, S D=2.06)$ and beverage $C$ laying in the middle $(M=4.54, S D=2.18)$. However, 
neither the main effect for color, $F<1$, nor the interaction between beverage and color was significant, $F<1$.

These findings clearly suggest that red color cues may reduce the amount of soft drink intake. Participants drank less from a cup with a red sticker compared to a cup with a blue sticker. Importantly, the effect of color on drink intake is not mediated by an increased liking for the drink. Rather, color seems to act as a direct signal on behavior without affecting perceived tastiness. These results lend first support to our hypothesis that red may act as a subtle stop cue and thereby influence consumption behavior.

\section{Study 2}

Results from Study 1 suggest that for drink intake the color red functions as a stop signal and thereby reduces consumption of soft drinks. Study 2 aimed to extend this finding to snacking behavior, and also included female participants. Moreover, because we were especially interested in incidental consumption (e.g., Wansink, 2004), Study 2 was implemented in a context where participants' focus of attention was directed away from the actual consumption behavior. Additionally, Study 2 was meant to rule out some potential alternative explanations. First, one might argue that because of associations with coolness and freshness, the color blue may be more suitable for a soft drink than the color red. Consequentially, a drink associated with blue may seem more desirable and hence consumption would be increased. Although we cannot rule out this account in Study 1, we consider it unlikely. Note that liking was not affected by the colors, as would be expected if blue indeed enhanced the appeal of the drink. Rather, we would like to argue that color directly signaled "approach" or "avoidance" without being mediated by an effect on the evaluation of the drink. Second, it is important to note that, in the current experiment, the absence of a proper control group renders it impossible to ascertain whether the effect at hand is predominantly driven by the color red signaling "avoidance", the color blue 
signaling “approach" (see, Mehta \& Zhu, 2009) or both. To address this constraint, we included a control group in Study 2, in which participants were exposed to white color.

\section{Method}

Participants. During a public information weekend of the local university 130 visitors (52 male, 72 female, 6 not reported) with a mean age of $31.68(S D=16.66)$ volunteered to participate in a short paper-pencil study.

Procedure and Materials. The study was set up in a booth where information about psychology was displayed. Visitors were invited to take part in a study related to "various areas of psychology". If they agreed they were given a stack of questionnaires, unrelated to the present purpose, and seated at a table where the experimenter had previously placed a paper plate containing exactly ten pretzels. Depending on the experimental condition, the background color of the pretzel plate was white, red or blue. Participants were invited to feel free to snack while they filled in the questionnaire. At the end of the questionnaire, participants were asked to evaluate the pretzels ("How much did you like the taste of the pretzels?") on a 7 -point scale $(1=$ not at all; $9=$ very much $)$ and to indicate the time of their last meal. After completion of the questionnaires, participants were told that the experiment was now over, probed for suspicions, debriefed and dismissed. Finally, the experimenter noted the number of eaten pretzels for each participant.

Because we used commercially available plates the color parameters (red: hue = 3 , saturation $=80$, lightness $=60$; blue: hue $=211$, saturation $=84$, lightness $=35$ ) differed from the ones used in Study 1, which had also been used by Mehta and Zhu (2009). Therefore, we conducted a pretest to ensure that the red and blue color used in Study 2 elicits similar associations as the colors used by Mehta and Zhu (2009) and in Study 1. Twenty-two participants were shown paper sheets of the two colors used in Study 1 and the two colors used in Study 2. Participants were asked to indicate on a 9-point scale 
ranging from 1 (not at all) to 9 (very much) how much several attention-related words applied to each color. The results indicate that the red color of Study 2 was more strongly associated with danger $\left(M_{\text {red }}=8.55, S D=0.51 ; M_{\text {blue }}=1.27, S D=0.63\right)$, stop $\left(M_{\text {red }}=8.45\right.$, $\left.S D=0.67 ; M_{\text {blue }}=1.68, S D=0.84\right)$, caution $\left(M_{\text {red }}=8.00, S D=0.97 ; M_{\text {blue }}=1.55, S D=\right.$ $0.80)$, and warning $\left(M_{\mathrm{red}}=8.32, S D=0.57 ; M_{\mathrm{blue}}=1.82, S D=1.22\right)$ than the blue color of Study 2 , all $p$ `s $<.001$. Moreover, neither the red nor the blue color differed in their associations between Study 1 and Study 2 (all $p$ `s $>.20)$.

\section{Results and Discussion}

None of the participants correctly guessed the purpose of the experiment. Twentyone participants were excluded from the analysis because they had shared their pretzels with friends or family resulting in a total of 109 participants (44 male, 61 female, 4 not reported) with a mean age of $31.80(S D=16.77)$ and a range from 13 to 75 .

To assess the experimental hypothesis that red inhibits snack food intake, we conducted a one-factorial (color condition: red vs. blue vs. white) ANOVA with the amount of eaten pretzels as dependent variable. The analysis revealed a significant effect of color condition on food intake $F(2,104)=3.00, p=.05, \eta_{\mathrm{p}}^{2}=.05$. Consistent with the predictions, planned comparisons revealed that participants in the red condition $(M=1.57$, $S D=2.29)$ ate significantly fewer pretzels than participants in the blue condition $(M=$ $3.00, S D=3.08), t(104)=2.17, p=.03, d=.52$ and participants in the white condition $(M$ $=2.91, S D=2.98), t(104)=2.04, p=.04, d=.50$. No significant differences emerged between participants in the blue condition and those in the white condition, $t(104)=.13, p$ $=.90$. To rule out a possible alternative explanation that differences in food intake were driven by differences in hunger levels, we repeated the ANOVA with participants' reported time of their last meal as a covariate. The differences in food intake still remained significant. 
A further analysis found no differences on the tastiness rating (all $p s>.14$ ) between the three plates, indicating that color did not affect participants` evaluation of the pretzels.

A possible alternative explanation might be that red plates may make food less appealing than blue or white plates. We addressed this concern in a further post-test. Twenty-eight participants were merely shown the plates with ten pretzels and rated each plate. Blue and red plates were liked equally and did not differ in respect to how appealing, appetizing, or attractive the food looked on them (all $p s>.26$ ). Compared to white plates red plates were liked more and were rated as even more appealing, appetizing, and attractive (all $p s<.001$ ), thereby ruling out the possibility that reduced food intake was elicited by lower appeal. In fact, participants ate more from the relatively unappealing white plate than from the more appealing red plate.

\section{General Discussion}

The present research investigated the role of color on snack food and soft drink intake. Consistent with our hypothesis, subtle red color cues significantly reduced the consumption of soft drinks (Study 1) and salty snacks (Study 2) compared to blue or white color cues.

We argue that red elicits avoidance motivation through its culturally learned and biologically embedded associations with danger, prohibition, and stop. It is important to note that red seems to act as a direct cue for consumption control without having an influence on consumption experience. In both studies evaluations of food or beverages were unaffected by color manipulations, indicating that red did not reduce the appeal of the consumed food or beverages.

Different from previous research, color was presented in a very subtle, unobtrusive way. Moreover, we tested our predictions not in a sterile lab, but at public places where participants were possibly distracted by many factors. In combination, these 
manipulations created situations most closely resembling natural situations of incidental consumption. Limitations and future research

In our studies color was a subtle cue in the environment rather than part of the food or drinks. Our research is not able to make a statement about how the color of food or beverages itself would influence the amount of food or beverages consumed. Prior research suggests that taste specific associations with color are very salient when consuming colored food (Shankar, Levitan, Prescott, \& Spence, 2009; Zampini, Sanabria, Phillips, \& Spence, 2007; Zellnera \& Durlach, 2002). In line with this research, the consumed amount of colored food and beverages may also - and possibly more strongly - be influenced by specific associations of color with taste (e.g., red berries are sweet, yellow lemons are sour etc.) than by more general associations (red lights, red stop signals, etc.).

Our manipulations were chosen to simulate naturally-occurring, incidental snacking and drinking behavior. Moreover, great care was taken to present the color cues outside of focused awareness. Our results would therefore suggest that the impact of color occurs at a non-conscious level. This assumption is also supported by the fact that nobody correctly guessed our hypothesis. However, future research may aim at further exploring the processes involved in color-induced consumption reduction and shed light on the role of consciousness in the impact of color.

Due to the increase in snack food and soft-drink consumption over the past few decades and the potentially detrimental consequences to people's health associated with this increase, we were primarily concerned with unhealthy food and drink consumption in our studies. However, an interesting question for future research may be whether the avoidance inducing effect of red would extend to healthy food and drinks as well. One could speculate whether the effect of red on food and drink intake may be diminished in a context where avoidance is not necessary (e.g., in the presence of healthy food, of which 
larger amounts can be consumed without causing negative effects). To date this remains an open question that should be addressed in future research.

Despite the need for more research in the domain of color and consumption behavior, we believe that the present research has direct implications for various domains concerned with snack and soft drink (and arguably other) consumption behavior. On the one hand, red color cues should be present when a reduction of unhealthy food or drink consumption is desired (for example when on a diet). On the other hand, red should be absent when a reduction of consumption is undesired (for example in a pub).

At present, we would be cautious to extend the findings to a clinical context as our participants were of non-clinical samples. However, our research suggests that the use of colors maybe a fruitful research topic with regard to the treatment of patients with certain eating disorders.

In sum, the present research offers a first step in understanding the subtle influences color can have on snack food and soft drink consumption. At present it is an open question how much variance color explains relative to other factors such as the presence of others (Pilner \& Mann, 2004), the size of food packaging (Rolls et al., 2004) or the size of food portions (Wansink, Painter, \& North, 2005). Yet, in light of the general increase in daily energy intake in the past years (Zizza, Siega-Riz, \& Popkin, 2001), we consider it important to explore all possible influences on consumption reduction and we believe that color is an important factor to consider when aiming to reduce consumption of unhealthy food and beverages. 


\section{References}

Bellizzi, J. A., \& Hite, R. E. (1992). Environmental color, consumer feelings, and purchase likelihood. Psychology and Marketing, 9, 347-363.

Elliot, A. J., \& Maier, M. A. (2007). Color and psychological functioning. Current Directions in Psychological Science, 16, 250-254.

Elliot, A. J., Maier, M. A., Binser, M. J., Friedman, R., \& Pekrun, R. (2009). The effect of red on avoidance behavior in achievement contexts. Personality and Social Psychology Bulletin, 35, 365-375.

Flegal, K. M., Carroll, M. D., Ogden, C. L., \& Curtin, L. R. (2010). Prevalence and trends in

obesity among US adults. 1999-2008. JAMA, 303, 235-241.

Gerend, M. A., \& Sias, T. (2009). Message framing and color priming: How subtle threat cues affect persuasion. Journal of Experimental Social Psychology, 45, 999-1002.

Herman, C., \& Polivy, J. P. (2004). The self-regulation of eating: Theoretical and practical problems. In R. F. Baumeister, \& K. D. Vohs (Eds.) Handbook of self-regulation:

Research, theory, and applications (pp. 492-508). New York: Guilford Press.

Khan, S. A., Levine, W. J., Dobson, S. D., \& Kralik, J. D. (2011). Red signals dominance in male rhesus macaques. Psychological Science, 22, 1001-1003.

Mehta, R., \& Zhu, R. (2009). Blue or red? Exploring the effect of color on cognitive task performances. Science, 323, 1226-1229.

Piernas, C., \& Popkin, B. M. (2010). Snacking increased among U.S. Adults between 1977 and 2006. The Journal of Nutrition, 140, 325-332.

Pliner, P., \& Mann, N. (2004). Influence of social norms and palatability on amount consumed and food choice. Appetite, 42, 227-237. 
Rolls, B. J., Roe, L. S., Kral, T. V. E., Meengs, J. S., \& Wall, D. E. (2004). Increasing the portion size of a packaged snack increases energy intake in men and women. Appetite, 42, 63-69.

Shankar, M. U., Levitan, C. U., Prescott, J., \& Spence, C. (2009). The influence of color and label information on flavor perception. Chemosensory Perception, 2, 53-58.

Tuomisto, T., Tuomisto M. T., Hetherington M., \& Lappalainen R. (1998). Reasons for initiation and cessation of eating in obese men and women and the affective consequences of eating in everyday situations. Appetite, 30, 211-222.

Van’t Riet, J., Sijtsema, S. J., Dagevos, H., \& De Bruijn, G.-J. (2011). The importance of habits in eating behavior. An overview and recommendations for future research. Appetite, 57, 585-596.

Wansink, B. (2004). Environmental factors that increase the food intake and consumption volume of unknowing consumers. Annual Review of Nutrition, 24, 455-479.

Wansink, B., Painter, J. B., \& North, J. (2005). Bottomless bowls: Why visual cues of portion size may influence intake. Obesity Research, 13, 93-100.

Zampini, M., Sanabria, D., Phillips, N., \& Spence, C. (2007). The multisensory perception of flavor: Assessing the influence of color cues on flavor discrimination responses. Food Quality and Preference, 18, 975-984.

Zellnera, D. A., \& Durlach, P. (2002). What is refreshing? An investigation of the color and other sensory attributes of refreshing foods and beverages. Appetite, 39, 185186.

Zizza, C., Siega-Riz, A. M., \& Popkin, B. M. (2001). Significant increase in young adults' snacking between 1977-1978 and 1994-1996 represents a cause for concern! Preventive Medicine, 32, 303-310. 


\section{Footnotes}

${ }^{1}$ We acknowledge that the meaning of color may vary depending on context. However, research by Mehta and Zhu (2009) suggests that red is most dominantly associated with danger or caution and less so with love or passion. A pretest $(\mathrm{N}=22$; see Study 2) conducted in our lab corroborates these findings. In absence of any specific context, the associations between red and danger, stop, caution and warning were significantly stronger than the associations between red and love, passion and romance, all $p$ 's $<.001$. 
Figure 1. Mean drink intake in $\mathrm{ml}$ for all drinks in Study 1. Standard errors are represented in the figure by the error bars attached to each column.

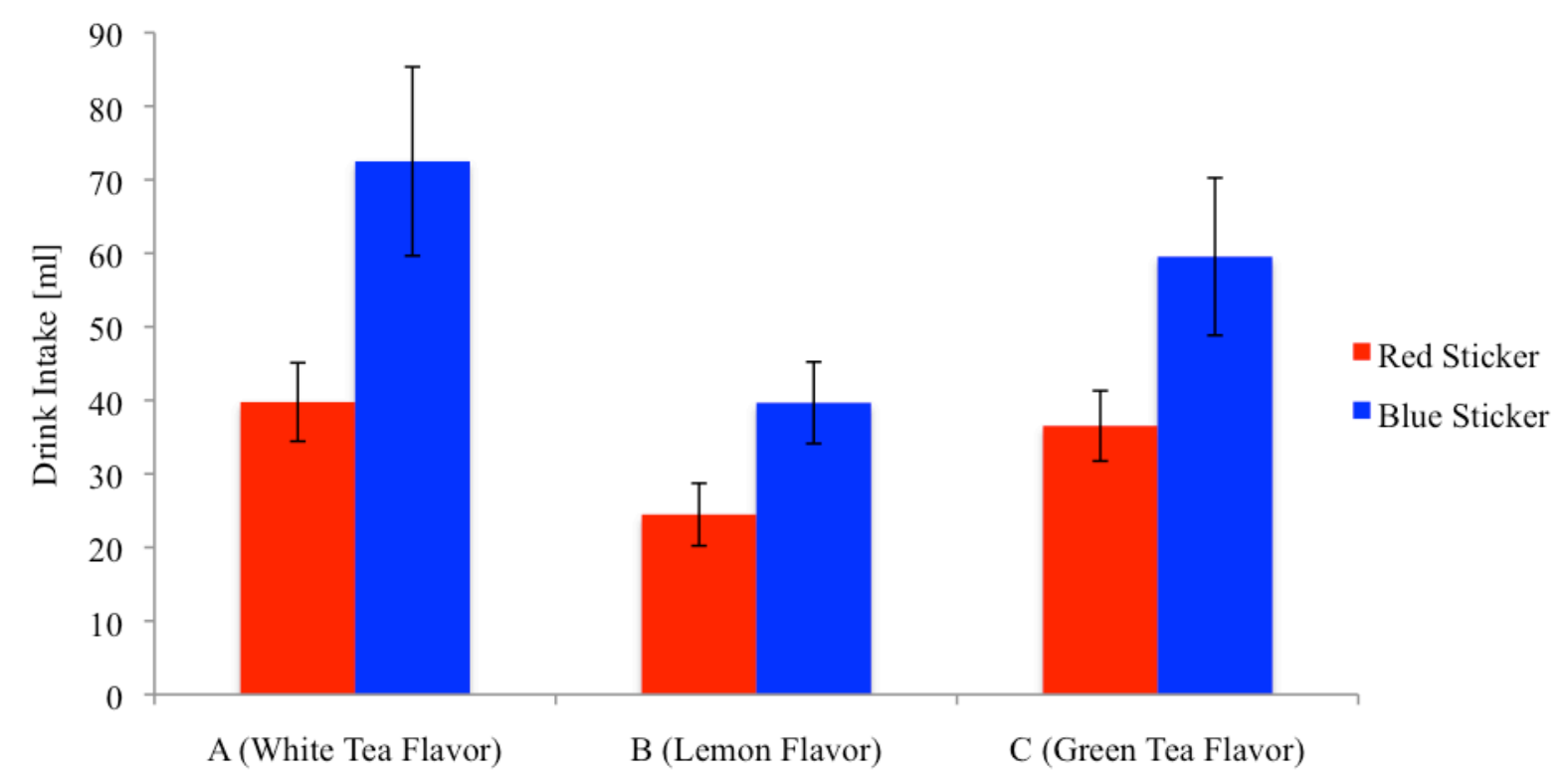

